\title{
CHARACTERISATION OF ROTARY KILN RESIDUES FROM THE PYROLYSIS OF SHREDDER RESIDUES: ISSUES WITH LEAD
}

\author{
Osric T. Forton ${ }^{a}$, Marie K. Harder ${ }^{*}$ and Norman R. Moles ${ }^{b}$

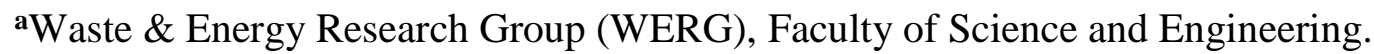 \\ University of Brighton. BN2 4GJ, UK \\ bApplied Geology Research Unit (AGRU), School of the Environment, University of \\ Brighton. BN2 4GJ, UK \\ *Corresponding author. Tel.: +44 1273 642539; fax: +44 1273642285. \\ E-mail address: m.k.harder@brighton.ac.uk (M.K. Harder).
}

\begin{abstract}
Stringent legislation has begun to be implemented across Europe relating to heavy metal contamination into the environment. This study thus focuses on developing a method for reliably determining the lead content of automotive shredder residue (ASR). The material is first pyrolysed to remove organic fractions. Different analytical methods were then used to investigate the concentrations of heavy metals in the burned char, which varies from chunks of metals in larger sized fractions to fine powders of mostly non-metals. By considering results from ICP-MS, EDXRF, WDXRF and a portable WDXRF, it was found that varying values were obtained but that consistent 'consensus values' could be determined. Such 'consensus' values of lead, copper, iron and zinc are thus reported, and show that properly depolluted ELVs have significantly lower lead levels than normal SR feed - 8000ppm versus $16000 \mathrm{ppm}$. The finest fraction, $<850$ microns, makes up around half of the mass of the SR and has only 2700ppm and 5400ppm lead concentration values for depolluted ELVs and normal SR respectively, making it of interest for further work to develop uses as a feed in other industries.
\end{abstract}

Author keywords: End-of-life vehicles, automotive shredder residue, ELV Directive, hazardous waste, pyrolysis, heavy metals, lead analysis 


\section{Introduction}

When ELVs are processed they are shredded into fist-sized pieces, and around $75 \%$ of the material is recovered as metal for the steel industry [1-4]. The remaining materials include foam, textiles, carpet, rubber, plastics, paint, wood and glass [3, 5-7] and are known collectively as automotive shredder residue (ASR). Commonly, shredder facilities process both ELVs and other feedstock (e.g white goods, light iron) together and the resultant waste stream is terms shredder residue (SR).

In most countries this material is disposed of to landfill, but this is considered unacceptable in the long term and mandatory requirements from legislative drivers have been introduced. The ELV Directive [8] requires a minimum of $85 \%$ material recovery from ELVs by 2015 - and only $75 \%$ is currently recovered with the ferrous and non-ferrous metals. This will be in addition to up to $10 \%$ energy recovery. This would mean that about $80 \%$ of the ASR volumes currently going to landfill will have to be recovered in the future. Commercial pyrolysis processing could do this if the char could be made useful, since it accounts for about 50\% the mass of the ASR (i.e. around 13\% of an ELV).[WERG, $2002 \ldots$ the Pyrolysis report]

Because this material is such a heterogeneous mixture, it is very difficult to produce a sample of less than one tonne of it that could confidently be called representative. For this reason, traditional analytical pyrolysis techniques are not used much for compositional analysis, because such methods often only involve samples of the order of a few grammes. As the char is a mixture of many different materials, some pieces weighing tens of grammes, it needs to be further size-reduced and thoroughly mixed in order to make suitable samples for analysis. However, it also contains metal pieces of varying sizes, which makes this very difficult for an analytical laboratory to process. Other studies have reported some metal levels $[5,11,12]$; they already show metals such as lead to be present at levels of some concern, and raise the question of whether new de-pollution techniques for ELVs can significantly reduce those levels.

In this paper larger quantities of ASR are pyrolysed, mixed until homogenous and analysed, and, making use of recent developments in X-ray fluorescent spectrometry, are shown to provide sound information for future standard analysis for ASR. This is an important developmental step, as other methods such as ICP-MS, AAS, AES and traditional XRF have significant difficulties analyzing this material. In fact, the difficulties to obtain a consensus between methods of the levels of metal contaminants is so great, that -making difficult for regulatory bodies [9]. A recent report by the UK Environment Agency highlighted the difficulty of determining the lead content in such a heterogeneous waste stream and declared this an ongoing problem. However, the results make considerable difference to the automotive shredder industry, which could potentially have their waste categorized as hazardous, requiring them to pay landfill disposal costs which make their businesses unviable. In this paper we present results to show that our method of using larger mixed samples and a new XRF is able to draw out consensus values. 
Using this improved method of analysis of ASR, the main focus of this paper is to develop work towards large-scale pyrolysis processes which could significantly change current practices. Many studies and processes have been published which report on potential pyrolysis of ASR, usually with a focus on producing useful gases or liquids such as diesels or oils $[2,3,5,6,10,11]$. Fewer have focused on the production of a char or ash suitable for use as a raw material $[5,11,12]$-though some have looked at ways of using it as a replacement fuel for fossil fuels [13]. However, despite this work, ASR is still not pyrolysed on a commercial scale, and this paper concentrates on one very significant barrier; the presence of heavy metals in the char which prevents it being used as a recycled material.

We report analytical results for 4 metals in the pyrolysed bulk samples of SR and ASR, namely lead, zinc, copper and iron. This paper thus helps to answer the following key questions, using ASR samples from the UK. What levels of lead and other heavy metals are actually in ASR - can a consensus value be obtained? How much difference would new practices, such as more thorough depollution of ELVs before shredding, or the removal of other scrap feedstock, make to these levels? Can simple post-pyrolysis processes such as sieving and crushing be developed to reduce metals in the char so that it can be used as a significant feed to industries such as cement and steel?

\subsection{Experimental Design \\ 2.1 Sampling and Sample Collection}

Two types of samples were pyrolysed. The first set, SR, was chosen as it reflects the type of feed mixture that is routinely dealt with at shredder plants in the UK - a mixture of ELVs, scrap iron and white goods. The effects of the general mixture of these three feeds will be seen in this sample, which is also typical of SR produced in the UK and currently landfilled.

The second type of samples, ASR, did not include waste other than ELVs, in order to accurately determine the metal levels due to ELVs alone. This information can be used by the automotive industry and its regulators to plan more recyclable and recoverable waste streams. All of these vehicles were depolluted to the new ELV Directive standards for this work [8], so that the samples would represent incoming new practices. This includes removal of the batteries, lead wheel weights, and fluids, all of which contribute lead [14, 15]. These ASR samples would thus not have heavy metal contributions from these, and thus would provide indications of the lowest realistically achievable contamination levels that can be achieved with the ELV types present in the waste stream today. This will be useful for the automotive industries to consider what further improvements could be developed for future cars.

Waste samples were collected in from a falling stream over 12 hours in quantities of one tonne and systematically coned and quartered [16-18] down to 10kg batches for the pyrolysis process [Williams et al, 2001]. The sampling of the depolluted ELVs was overseen particularly carefully to ensure that it did not contain any other feed.

\subsection{Pyrolysis reactor and process}

The pyrolysis process used in this work is basic, and can easily be reproduced by others. The main difference to most work reported is that the pyrolyser was able to process 
samples of 50kg (using continuous feed), rather than the few grammes reported in some other studies [19]. A continuous rotary kiln was used in which the samples were fed into the reactor at a rate of $1 \mathrm{~kg} / \mathrm{min}$ and pyrolysed at $650^{\circ} \mathrm{C}$ for a residence time of about 12 mins. (Continuous mode has many commercial advantages, but the results presented here can be equally well reproduced in batch mode). The rotary reactor was $480 \mathrm{~mm}$ long and $180 \mathrm{~mm}$ in diameter. It was externally heated using propane gas. A typical pyrolysis run lasted 15min, of which 12 mins were spent in the central reactor space at the required temperature and the rest in approaching and falling away. The solid residues were the collected for further post-pyrolysis processing. The same pyrolysis conditions were used for both types of waste streams studied. Specific details of the pyrolysis process have previously been reported in $[12,20]$.

\subsection{Characterisation of the pyrolysed char}

Both ASR and SR residues were analysed for heavy metal concentrations. Analysis using several techniques was carried out in order to arrive a 'consensus values' that could be considered robust. Results for of zinc, iron, copper and lead are reported here.

From previous studies it was expected that levels of contaminant metals $[14,15]$ would be higher than industry would accept. Some experimentation was thus carried out in this work with the char separated into different sized fractions and processed to see if some combination of post-pyrolysis crushing and sieving could concentrate the remaining contaminant metals into one or two fractions, leaving the rest less contaminated. Before such sieving could be carried out, it was first necessary to crush the raw char to separate off any pieces of metal for recycling, as would happen in industry. An elemental analysis was then carried out on each of the fractions to find out how they varied.

The details of the methods used in the post-pyrolysis processing, the analysis of heavy metals and the obtaining of consensus values are given below.

\subsubsection{Post-Pyrolysis Crushing and Sieving Fractions (mechanical separation and roll crushing of char)}

A set of mechanical roll crushing, screening and particle size separation procedures were adopted in order investigate recoverable materials and contaminants in specific size fractions (Fig. 1). For both the ASR and SR waste streams, the process involved an initial screening at $2000 \mu \mathrm{m}$ in order to remove residual ferrous metals in the form of large irregular pieces. This step parallels that likely to take place on a commercial site, where the larger pieces of metal will be removed. The continuing processing of the $\mathrm{s}<2000 \mu \mathrm{m}$ particles was labeled Leg A, and that of the s $>2000 \mu \mathrm{m}$ particles was labeled Leg B.

Both streams were then crushed, to remove the carbonaceous residues from smaller pieces of metal. In particular, roll crushing was used to try to increase the effective size of the soft lead and copper metals by flattening them. The remaining char was sieved at different fractions of $2000 \mu \mathrm{m}, 1400 \mu \mathrm{m}, 850 \mu \mathrm{m}$ and $500 \mu \mathrm{m}$. This allowed the relative metal contents in each fraction to be investigated, which also was expected to assist in the determination of their source. For example, lead possibly present as a residue from old- 
fashioned leaded petrol, or from battery fluids, would be expected to be in the finest fractions whilst slivers of metallic lead knocked off larger pieces would be expected in larger sized fractions.

In fact, one further step was introduced into the process, to ensure that the entire sample was not contaminated from potential fine lead dust. Before the crushing took place, the samples were sieved at $850 \mu \mathrm{m}$ so that any such dust would be removed rather than then mixing in with all daughter samples. This was only relevant for Leg A in each case.

[Insert Fig. 1]

\subsubsection{Chemical analyses of the char - obtaining 'consensus values'}

The chemical analysis of waste streams such as ASR or SR is difficult. This is for several reasons. One is the very large number of elements and species present in this complex waste. Any form of spectroscopy will show up dense populations of lines which challenge fitting routines. In X-ray techniques, it is possible for X-rays to be absorbed and re-emitted from some elements and then interfere with other elements - a matrix effect. Without calibration standards for such a complex material, this effect cannot be overcome to allow proper calibration. Thus, no single analytical method can claim to provide excellent results for ASR or SR - and results from different methods can give different absolute answers. In dry solid sample preparations it is difficult to grind the sample finely due to metal and foam pieces. In dissolution preparations the larger pieces may not dissolve fully or proportionately.

An important outcome of the work presented in this paper is the derivation of 'consensus' values for metal levels in ASR, which are presented after considering the performance of several analytical methods on different sized fractions. By comparing results of different analysis techniques on some fractions, it was possible to gain an overview of their strengths and weaknesses, which was subsequently useful for fractions where only one technique was available (e.g. Fig. 1 for $\mathrm{Pb}$ ). In this manner 'consensus values' were derived for all of the post-pyrolysis fractions.

In each case, the carbon in the char was removed by ashing at $550^{\circ} \mathrm{C}$ (burning off all of the carbon) for a set of 6 sub samples from each waste stream. Then, some samples were analysed with ICP-MS analyses after acid dissolution [21] (labeled as ICP-MS in Fig. 2). Analyses of some other samples used various solid media X-ray fluorescence (XRF) methodologies [22] (labeled as WDXRF1, WDXRF2 and PXRF in Fig. 2). The WDXRF1 samples were analysed as pressed pellets using a Panalytical ${ }^{\circledR}$ MAGIX-Pro 4kW WDXRF using an IQ+ software package based on an advanced fundamental parameter algorithm for matrix correction. The WDXRF2 samples were analysed as fused beads using a Philips ${ }^{\otimes}$ PW1480 WDXRF instrument calibrated with custom made synthetic standards made of reagent grade elements. Lastly, the PXRF samples were analysed as pressed powder pellets in quintets using Innov- $\mathrm{X}^{\oplus}$ alpha 6500 analyzer employing a $35 \mathrm{kV} \mathrm{Ag}$ anode $\mathrm{X}$-ray tube with a Compton normalization [23] procedure for both matrix effects and background correction [24]. The PXRF analysis included multiple measurements, but all others were carried out by commercial labs and only reported single measurements, hindering the calculation of the inherent uncertainty in the measurements for them.

As noted, these various techniques were suited to different fractions of the ASR, sometimes overlapping. Overall consideration of them all was expected to lead to a 
'consensus value' for each of the metal contents.

\section{Results}

\subsection{Consensus of values}

Figures 2 - 5 below show the results returned by the various analytical methods for different fractions. It was not appropriate to use any one technique for all fractions, but there is some overlap of the methods for some samples. Individual trends peculiar to each of the methods can be seen, but the point being made here is that by displaying these all together, an estimate can be made of a calue for the metals present.

\section{[Insert Fig. 2 - 5]}

For each fraction and each metal, the 'consensus' value is provided, and all are summarized in Table 1. These are not proper averages, as it would be necessary to have uncertainties for each method and value in order to weight the different methods properly for an average, and in most cases the laboratories did not provide these. For example, in Fig. 2 for Pb, it can be seen that the WDXRF2 method often gives lower values - and is in fact operating at its lowest detection limit of 1000ppm even though the PXRF and WDXRF methods report values well above this limit. However, whenever the values are over 2000ppm, the fused bead results are comparable with the others. The WDXRF1 and PXRF results are closely similar, except for high concentrations of $\mathrm{Fe}$, where they start to differ.

A very important contribution of the above work is that the resultant figures are reasonably robust, and not subject to the peculiarities of a given analytical method, because they use 'consensus values', consistent with 3-4 independent analytical methods. The results from the different fractions can be summed to produce overall figures for metal contents in both SR and ASR samples that can be further discussed.

\subsection{Values for overall metal levels in ASR and SR}

Table 2 below summarises the levels of metals present in the overall char. It clearly shows that lead levels in the ASR samples, which only contained de-polluted ELVs, are significantly less that the more typical SR samples - almost by a factor of 2 . This is very encouraging for both the responsible producer - the automotive industry - and prospective users of the char as a raw material. However, to avoid being classified as a hazardous waste it is necessary in California for materials to have less than 1000ppm of lead [25]. Thus, the data shows that, even with stringent application of the new de-pollution requirements, ASR has levels above that important threshold. This is the first time that these levels have been reported with such confidence. The implications for the industries are significant.

\section{[Insert Table 2]}

The data also indicates that either the depollution of the cars has significantly reduced the amounts of lead in ASR, or the other feedstock materials to SR, namely white goods (such as ovens and washing machines) and light iron (such as bicycles and fences), are contributing significant amounts of lead. Previous work has suggested that there is little difference between ASR with and without the extra depollution [26], therefore it seems possible that the additional $\mathrm{Pb}, \mathrm{Zn}$ and $\mathrm{Cu}$ observed in SR samples derives from white 
goods and light iron feedstock. This is obviously a preliminary result, but one which merits further investigation by, for example, considering shredded samples of white goods on their own.

\subsection{Variations between different post-pyrolysis crushed and sieved size fractions}

Figs 2 -5 indicate variations in different fractions, after the milling and processing described in 2.3. Besides their use for providing the 'consensus' values discussed in 3.1, these figures show important characteristics of the sub-streams of this process. For example, consider the lead distributions in the $<2 \mathrm{~mm}$ Legs in Fig. 2. For the ASR the levels of lead do not go over 4000ppm except for one fraction - the 1.4-2.0 mm (Fig. 2, Pb ASR_Leg A).

However, the large level present is not seen in the same fraction originating from larger pieces, which started off as $>2 \mathrm{~mm}$ in the char (Fig. 2, Pb ASR_Leg B). It could originate from fillers or additives in plastics, or chips of paint. Whatever its source, it is clearly an automotive component or ingredient, as there is no correspondingly high value for the SR samples.

The patterns of lead in different fractions are significantly different for the two samples, SR and ASR. On the other hand, they are consistent, in that the ELVs in the ASR sample would be expected to normally account for no more than $40 \%$ of the feed of SR, and this is supported in that none of the levels seen from the ASR are exceeded in the SR. Thus, the large levels (7000ppm) of lead in the 1.4-2.0 mm ASR fraction would contribute to levels of less than 2.5 times this in the SR; this is seen, as the levels are only $2500 \mathrm{ppm}$. In this manner it can be shown that most of the lead in the larger sized fractions of the SR can be accounted for by the ELVs; the exceptions are the categories $<1.4 \mathrm{~mm}$, where the lead must be coming from non-depolluted ELVs or the other SR components.

The copper values tell a different story. Levels exceeding $60000 \mathrm{ppm}$ (i.e. 6\%) are seen in both ASR and SR samples in the larger sized fractions, so it must be concluded that the light iron and white goods are contributing more than the ELVs. The exception is the 0.85$1.4 \mathrm{~mm}$ fraction (Fig. 4, Cu SR_Leg A) where the ELVs make a strong contribution almost certainly due to copper wiring.

\subsection{Discussion and Conclusions}

A previous study on pyrolysis products of ASR from Europe and Canada, [2] also showed that the metals and inorganic materials in the feedstock are concentrated in the solid residues. Manual, magnetic and mechanical particle size (s) separations were used $(\mathrm{s}<125 \mu \mathrm{m},<250 \mu \mathrm{m},<850 \mu \mathrm{m},<2000 \mu \mathrm{m}$ and $<6400 \mu \mathrm{m})$ respectively to recover large metallic pieces, ferrous metals and inorganic material in each size fractions. An Aqua Regia digestion followed by ICP-AES analysis of the different fraction showed that proportion of metals including $\mathrm{Pb}, \mathrm{Zn}, \mathrm{Cu}$, Fe varied with particle size. $\mathrm{Pb}$ and $\mathrm{Zn}$ were uniformly distributed in all size fractions below $2 \mathrm{~mm}(\sim 0.4 \mathrm{wt} \%)$ while $\mathrm{Cu}$ was concentrated in the size range $6.4-0.5 \mathrm{~mm}$. The study showed that it was possible to recover about $14 \%$ by weight of useful metals remaining in the solid residues [2] characterised the solid residues resulting from a commercial screw kiln pyrolysis of ASR and showed the levels of $\mathrm{Zn}, \mathrm{Pb}$ and $\mathrm{Cu}$ were particularly high in fine and coarse fractions after particle size separation. The high levels of $\mathrm{Cu}$ were attributed to the presence of electric copper wire in the fractions. 
Elemental analyses of ASR and SR have been presented here to investigate several questions. First, it was necessary to bring together measurements of the same material from different analytical techniques in order to present a 'consensus' value in each case that could be considered useful. This was successful done, by comparing the techniques across a range of samples with quite different complex combinations of elements, e.g. copper ranging from $1 \%$ to $8 \%$; zinc from $0.5 \%$ to $12 \%$. This resulted in levels of the four metals being reported with confidence for both the ASR and SR overall.

The overall values for lead in ASR were reported at 2400ppm and for SR at 6220ppm. These values are very useful, as they indicate a clear reduction for ASR (using de-polluted ELVs) from the typical SR. However, the values are still above the 1000ppm threshold that is important in the consideration of hazardous materials, and shows the need for further work to reduce the lead levels. 


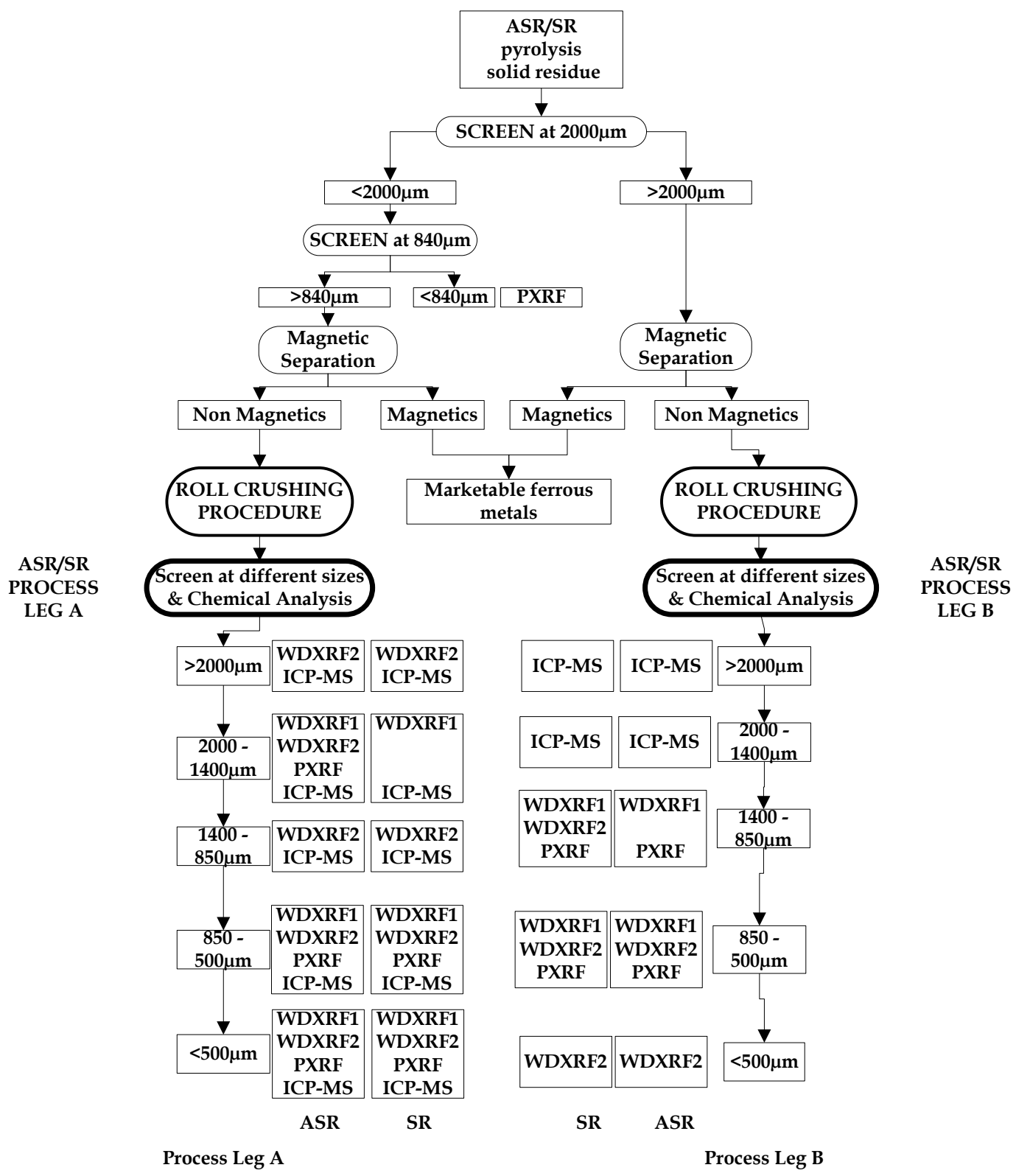

Fig. 1: Schematic of the mechanical screening and roll crushing methodology adopted for both SR and ASR, and analytical methods used on specific samples in Process Legs A and $\mathrm{B}$ and for $<\mathbf{8 5 0} \boldsymbol{\mu m}$. (Note that samples analysed by different methods from the same sub sample are shown as insets in the boxes next to each particle size. ASR and SR samples analysed by specific methods are also shown. These samples are directly comparable with variations being a function of method performance while variations between boxes /particle sizes are a function of the ability of the mechanical process to concentrate the metals in specific size fractions. Relative trends in the data sets are presented in the text) 

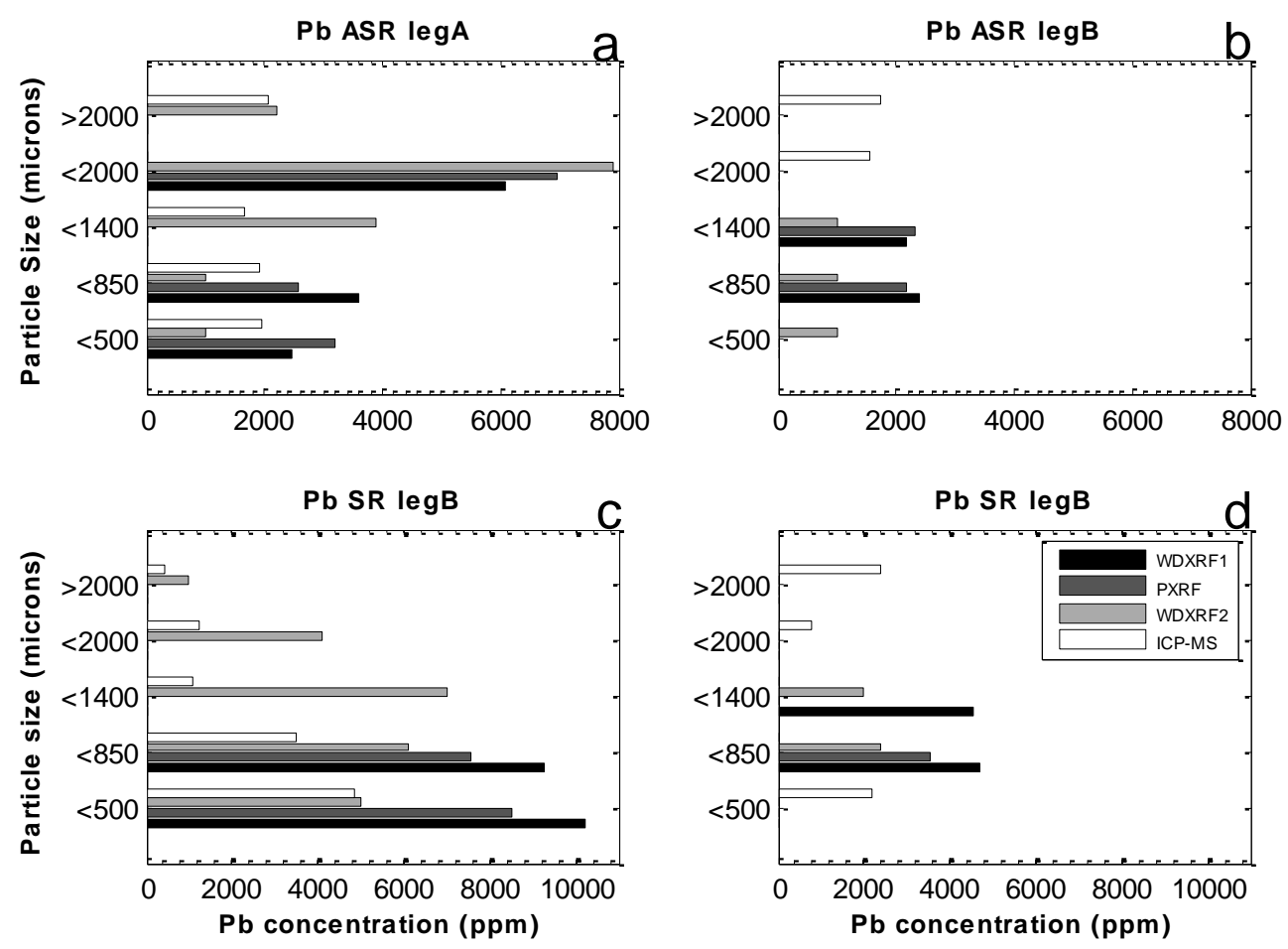

Fig. 2: Relative concentrations of $\mathrm{Pb}$ in different particle sizes of ASR and SR by different analytical methods 

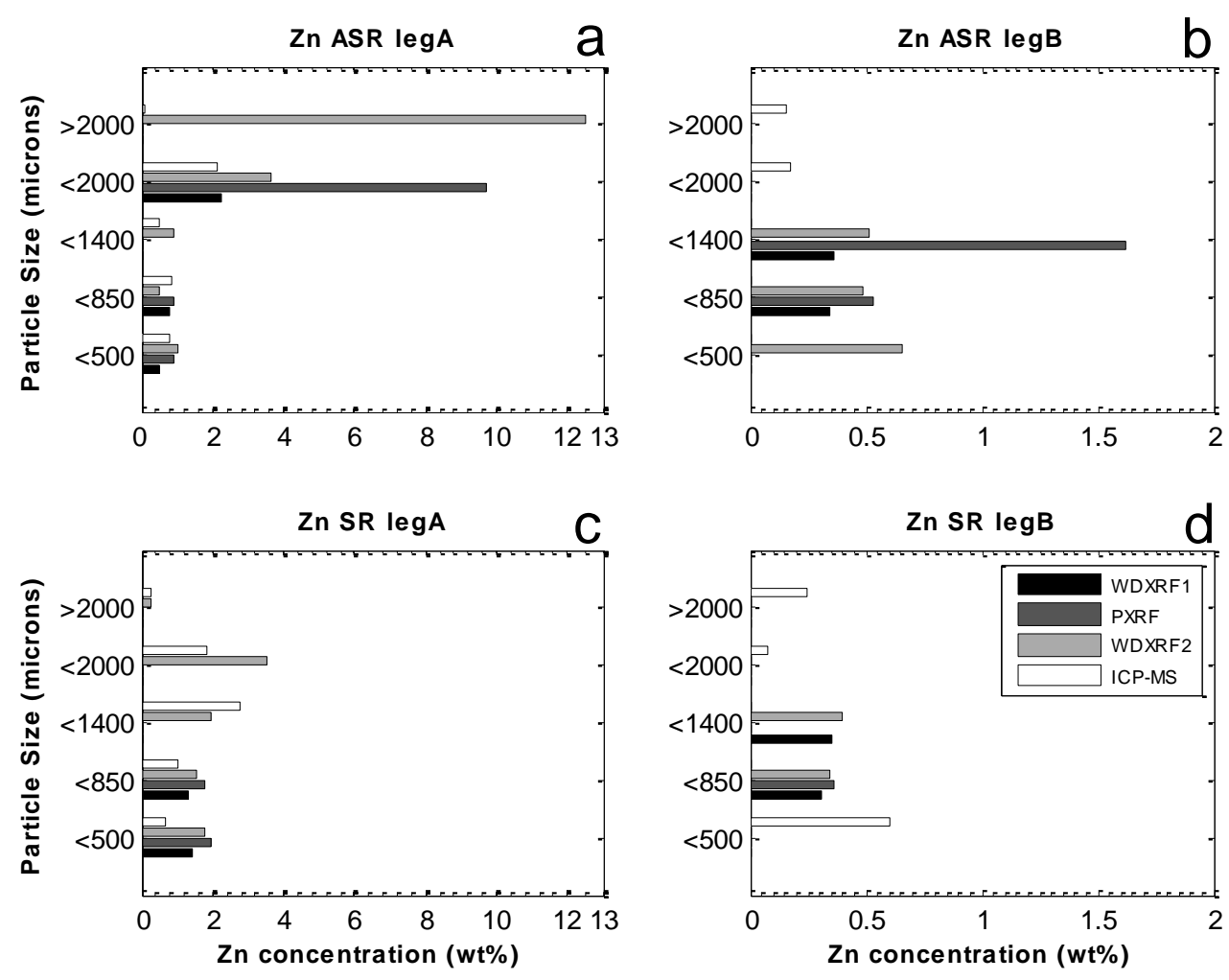

Fig. 3: Relative concentrations of $\mathrm{Zn}$ in different particle sizes of ASR and SR by different analytical methods 

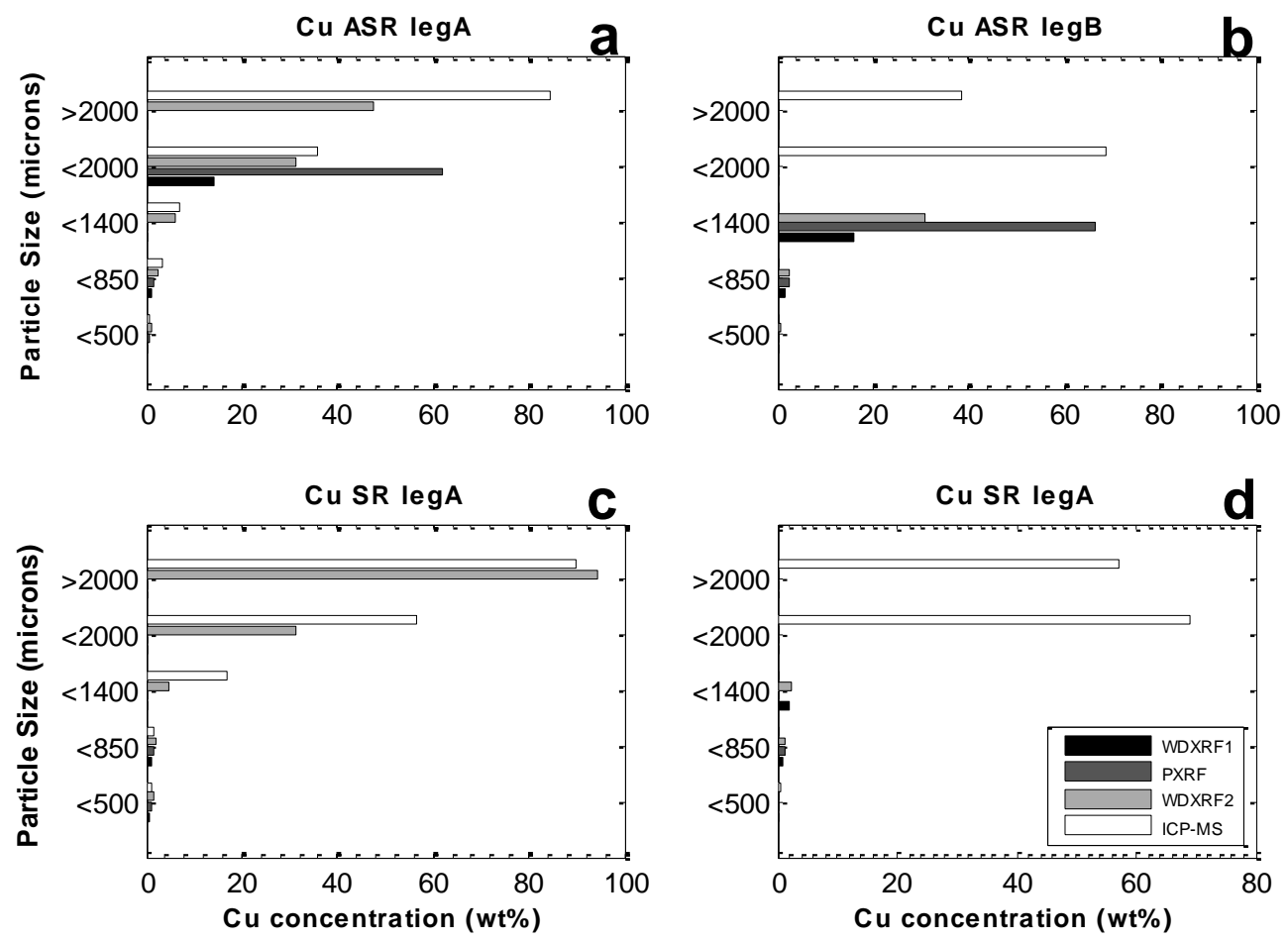

Fig. 4: Relative concentrations of $\mathrm{Cu}$ in different particle sizes of ASR and SR by different analytical methods
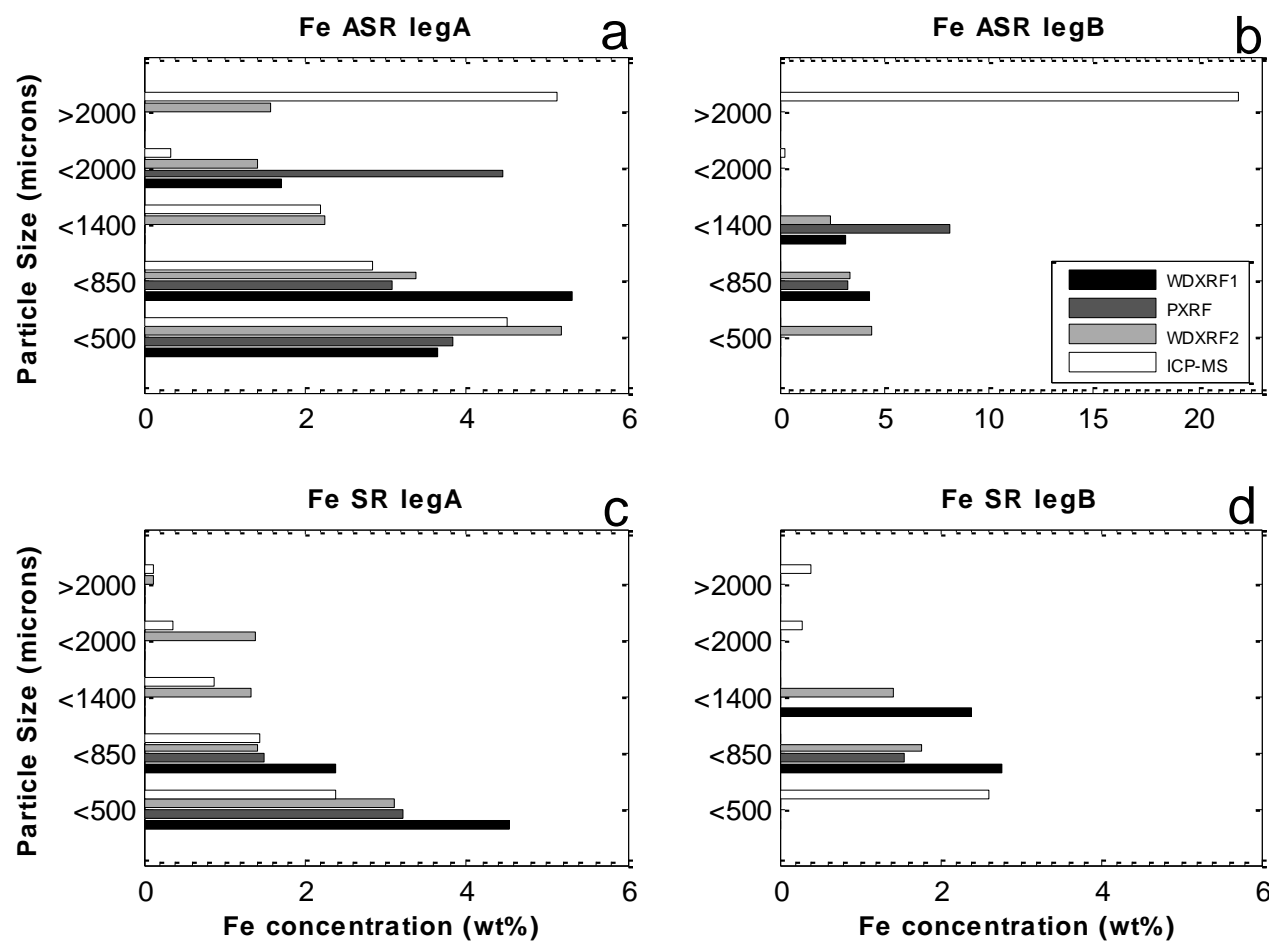

Fig. 5: Relative concentrations of Fe in different particle sizes of ASR and SR by different analytical methods 
Table 1: Consensus values for levels of $\mathrm{Pb}, \mathrm{Zn}, \mathrm{Cu}$ and $\mathrm{Fe}$ in ASR and SR char residues (all values are presented in ppm)

\begin{tabular}{lccccccccc}
\hline & Material Type & SR & ASR & SR & ASR & SR & ASR SR & ASR \\
\hline Process & & & & & & & & & \\
Leg* & Particle size/mm & Pb & Pb & Zn & Zn & Cu & Cu & Fe & Fe \\
\hline Leg A & $<850 \mu \mathrm{m}$ & 5336 & 2077 & 12168 & 7645 & 2093 & 1244 & 40089 & 36468 \\
Leg A & $>2000 \mu \mathrm{m}$ & 1 & 1 & 4 & 21 & 1332 & 219 & 2 & 11 \\
Leg A & $1400-2000 \mu \mathrm{m}$ & 13 & 24 & 131 & 184 & 2142 & 1242 & 43 & 88 \\
Leg A & $850-1400 \mu \mathrm{m}$ & 123 & 75 & 722 & 253 & 3296 & 1603 & 336 & 610 \\
Leg A & $500-850 \mu \mathrm{m}$ & 154 & 49 & 329 & 139 & 404 & 364 & 517 & 679 \\
Leg A & $<500 \mu \mathrm{m}$ & 236 & 82 & 478 & 240 & 379 & 201 & 957 & 1294
\end{tabular}

Non magnetic fraction $>\mathbf{2 m m}$ process leg $(\operatorname{Leg} B)$

\begin{tabular}{cccccccccc} 
Leg B & $>2000 \mu \mathrm{m}$ & 54 & 13 & 34 & 13 & 14230 & 2791 & 90 & 1596 \\
Leg B & $1400-2000 \mu \mathrm{m}$ & 9 & 6 & 6 & 7 & 9442 & 2447 & 36 & 9 \\
Leg B & $850-1400 \mu \mathrm{m}$ & 106 & 47 & 97 & 194 & 790 & 7964 & 612 & 960 \\
Leg B & $500-850 \mu \mathrm{m}$ & 85 & 72 & 84 & 159 & 276 & 658 & 487 & 1142 \\
Leg B & $<500 \mu \mathrm{m}$ & 104 & 0.0075 & 57 & 75 & 241 & 75 & 1225 & - \\
\hline
\end{tabular}

* as shown in Fig. 1

Table 2: Overall metal levels present in both Automotive Shredder Residue and Shredder Residue sample chars

\begin{tabular}{lllll}
\hline $\begin{array}{c}\text { Sample Type/ Elemental } \\
\text { concentration (ppm)* }\end{array}$ & Pb & $\mathbf{Z n}$ & $\mathbf{C u}$ & $\mathbf{F e}$ \\
\hline $\begin{array}{l}\text { Shredder residue (mixed light } \\
\text { iron, white goods and ELVs }\end{array}$ & 6220 & 14108 & 34624 & 44393 \\
$\begin{array}{l}\text { Automotive shredder Residue } \\
\text { (depolluted ELVs only) }\end{array}$ & 2446 & 8930 & 18809 & 42856 \\
$\begin{array}{l}\text { *These values were obtained by summing the elemental values of the different } \\
\text { fractions as presented in Table 1. }\end{array}$ & & & &
\end{tabular}

\section{References}

[1]S. Das, T.R. Curlee, C.G. Rizy, and S.M. Schexnayder. Res. Con. Recyc. 14 (1995) 265 [2]M. Day, J.D. Cooney, and Z. Shen, J. Anal. Appl. Pyrolysis. 37 (1996) 49

[3]M. Zolezzi, C. Nicolella, S. Ferrara, C. Iacobucci, and M. Rovatti, Waste Man. 24(2004) 691

[4]O. T. Forton, M.K. Harder, and N.R. Moles, Res. Con. Recyc. 46 (2006) 104

[5]M. Day, Z. Shen, and J.D. Cooney, J. Anal. Appl. Pyrolysis. 51 (1999) 181

[6]S. Galvagno, F. Fortuna, G. Cornacchia, S. Casu, T. Coppola, and V.K. Sharma, Energy. Conv. Man. 42 (2001) 573

[7] R. Rausa and P. Pollesel, J. Anal. Appl. Pyrolysis. 40-41 (1997) 383

[8] European Commission, Directive 2000/53/EC of the European Parliament and of the 
Council of 18 September 2000 on end-of-life vehicles. Official Journal of the European Communities. 2000, European Commission. 2000

[9] Environment Australia, Environmental Impact of End-of-life Vehicles: An Information Paper. Australian Government Department of the Environment and Heritage. ISBN 0642547513. 2002

[10] A. Chaala, O.G. Ciochina, and C. Roy, Res. Con. Recyc.26 (1999) 155

[11]C. Roy and A. Chaala, Roy, Res. Con. Recyc. 32 (2001) 1

[12]WERG, Pyrolysation of shredder residue (fines). A report by the Waste \& Energy Research Group, University of Brighton, commissioned by the Brighton Environmental Body Ltd for the Consortium of Automotive Vehicle Recycling. 2002: Brighton, UK. 2002

[13]B. Boughton, Evaluation of shredder residue as cement manufacturing feedstock. 2006, California Environmental Protection Agency, USA.

[14]J. Lohse, K. Sander, and M. Wirts, Heavy metals in Vehicles II. Final Report. OKOPOL. 2001: Hamburg. 2001

[15]K. Sander, J. Lohse, and U. Pirntke, Heavy Metals in Vehicles. Final Report . OKOPOL. 2000: Hamburg. 2000

[16] EA and BMRA, Protocol for the sampling and preparation of shredder residues for analysis. 1997: Bristol, UK. 1997

[17] Perry, D. and J. Thompson, Inst. of Waste Man. Proc. 1998, pp 4 -7

[18] M. Fingas, K. Li, N. Laroche, P. Jokuty, C. Brown, and B. Fieldhouse. Proc. sixteenth technical seminar on chemical spills. 41-45. Ottawa, Canada. 1999.

[19].D. Lanoir, G. Trouve, L. Delfosse, D. Froelich, and A. Kassamaly, Waste Man. Res. 15 (1997) 267

[20]K. S. Williams., L. McGrady, and M.K. Harder. Pyrolysis of shredder residue to recover valuable material. in Proc. Inst. of Mech. Eng. Sixth International Conference on Engineering for Profit from Waste. 2001. London, UK.

[21] E. Prichard, G.M. MacKay, and J. Points, Trace Analysis: A structured approach to obtaining reliable results. 1996, Cambridge, UK: The Royal Society of Chemistry

[22]E. P. Bertin, Principles and Practice of X-Ray Spectrometric Analysis. 2nd ed. 2 ed. 1979, New York: Plenum Press.

[23]L. Broothaers, Spectrochim. Acta. 34 (1979) 177

[24]Y. Hua and C.T. Yap, X-Ray Spec. 23 (1994) 27

[25]P. J. Wood. California's Automobile Shredder Waste Initiative. Draft Report. 2002, Department of Toxic Substances Control: Sacremento.

[26]WERG, Improving Lead levels in Automotive Shredder Residue. A report by the Waste and Energy Research Group, University of Brighton, commissioned by Brighton Environmental Body Ltd for the Consortium of Automotive Vehicle Recycling. 2005 